\title{
Brain SPECT Imaging with Acetazolamide Challenge*
}

\author{
Mickaila Johnston \\ Division of Nuclear Medicine, Department of Radiology, University of Arkansas for Medical Sciences, Little Rock, Arkansas
}

$\mathbf{T}$ he patient was a 69-y-old man who, $37 \mathrm{~d}$ earlier, had a 4-d-long evolving onset of headache, dizziness, acute personality changes, visual changes, and weakness. Unspecified imaging was obtained at an outside facility, and he was diagnosed with an intracerebral stenosis. Subsequently, he was given aspirin and clopidogrel (Plavix; Bristol-Myers Squibb/Sanofi Pharmaceuticals Partnership) and told to return for reevaluation in $1 \mathrm{mo}$. At the 1-mo follow-up, his physical examination was interpreted as being significant for a Glasgow Coma Scale score of 15, and cranial nerves were intact. His examination was otherwise effectively unremarkable. Laboratory findings were normal. The nuclear medicine department was consulted because there was concern about possible intracerebral stenosis.

A 2-d ${ }^{99 m}$ Tc-exametazime $(1,184$ and $1,073 \mathrm{MBq}[32$ and $29 \mathrm{mCi}$, respectively) baseline SPECT/CT scan, followed $24 \mathrm{~h}$ later by a 1-g acetazolamide (Diamox; Teva Pharmaceuticals) challenge SPECT/CT scan, was obtained to evaluate for cerebrovascular abnormalities. A non-contrastenhanced CT scan was acquired for attenuation correction using a 5-mm slice interval, a low-energy all-purpose filter, a zoom of 2.19 , a $273 \times 273$ field of view, and a $128 \times 128$ matrix. Emission imaging was obtained with 128 angles, at $25 \mathrm{~s}$ per step. Data were processed using ordered-subset expectation maximization (4 iterations, 8 subsets) and a Butterworth filter (cutoff, 2.0; order, 2.53). No sedatives were administered.

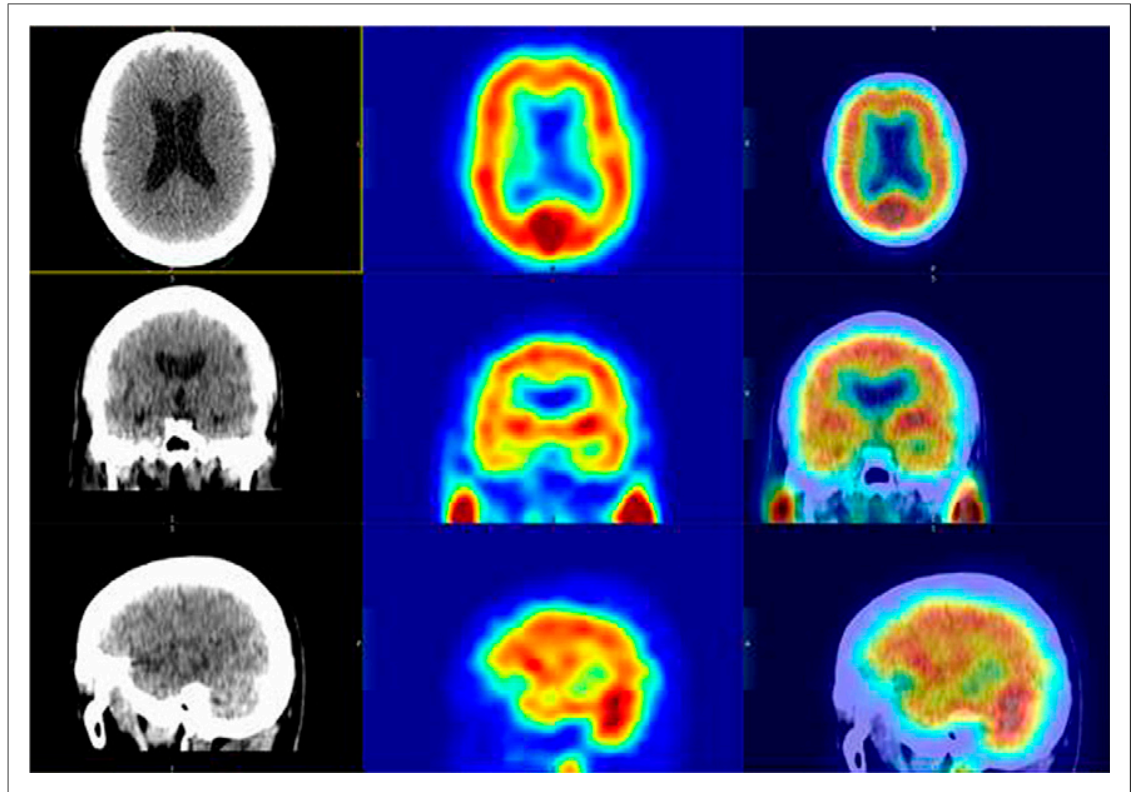

FIGURE 1. Combined CT, SPECT, and fusion images (axial, coronal, and sagittal) performed under baseline conditions.

On challenge imaging, in comparison to baseline, there was a 30\% decrease in radiotracer distribution involving the left cerebral hemisphere. A 30\% decrease was calculated by comparing the uptake in the noneffected hemisphere and within the effected hemisphere on a 20 -step color scale (where each shade represents a 5\% change). A direct comparison was made by counting how many color shades of difference there was and correlating that to the total number of shades available (e.g., there were 6 shades of color difference, on a 20-step scale, which results in a $30 \%$ difference). No significant motion was appreciated, and the quality of the images was considered technically adequate. This finding was consistent with a positive acetazolamide-enhanced brain perfusion study, indicating a significant lack of flow reserve to the left cerebral hemisphere.

Neurointerventional radiology identified a high-grade stenotic segment of the left supraclinoid ICA measuring approximately $16 \mathrm{~mm}$ in length, with a significant flow limitation (Fig. 1A). Successful balloon angioplasty of the left supraclinoid ICA was performed (Fig. 1B). The patient remained on clopidogrel and aspirin, to be followed with contrastenhanced magnetic resonance angiography after 3 mo.

Published online Feb. 12, 2013.

COPYRIGHT (C) 2013 by the Society of Nuclear Medicine and Molecular Imaging, Inc.

DOI: $10.2967 /$ jnmt.112.118778 


\section{QUESTION 1}

Which of the following is a known contraindication to an acetazolamide study?

A. A documented sulfonamide allergy.

B. A history of acute mountain sickness.

C. A stroke 3 wk previously.

D. Renal insufficiency.

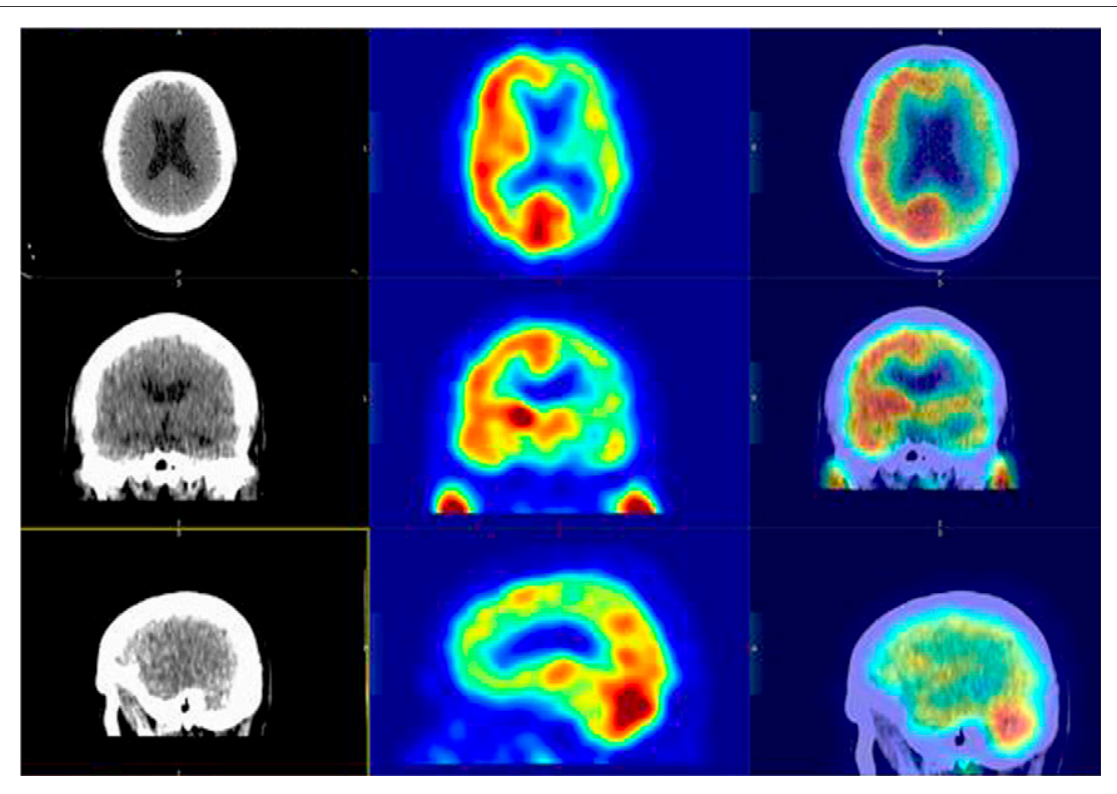

FIGURE 2. Combined CT, SPECT, and fusion images (axial, coronal, and sagittal) performed under acetazolamide challenge line conditions.

\section{QUESTION 2}

Concerning Figure 2 (imaging performed under baseline conditions) and Figure 3 (during acetazolamide challenge), which is the most accurate interpretation?

A. Normal findings.

B. Equivocal findings.

C. Abnormal findings with an area of hyperperfusion within the right parietal lobe.

D. Abnormal findings with an area of hypoperfusion within the left cerebral hemisphere.

\section{QUESTION 3}

Considering the best answer for Question 2, which vessel is most likely involved?

A. Left internal carotid.

B. Right internal carotid.

C. Left vertebral artery.

D. Right vertebral artery.
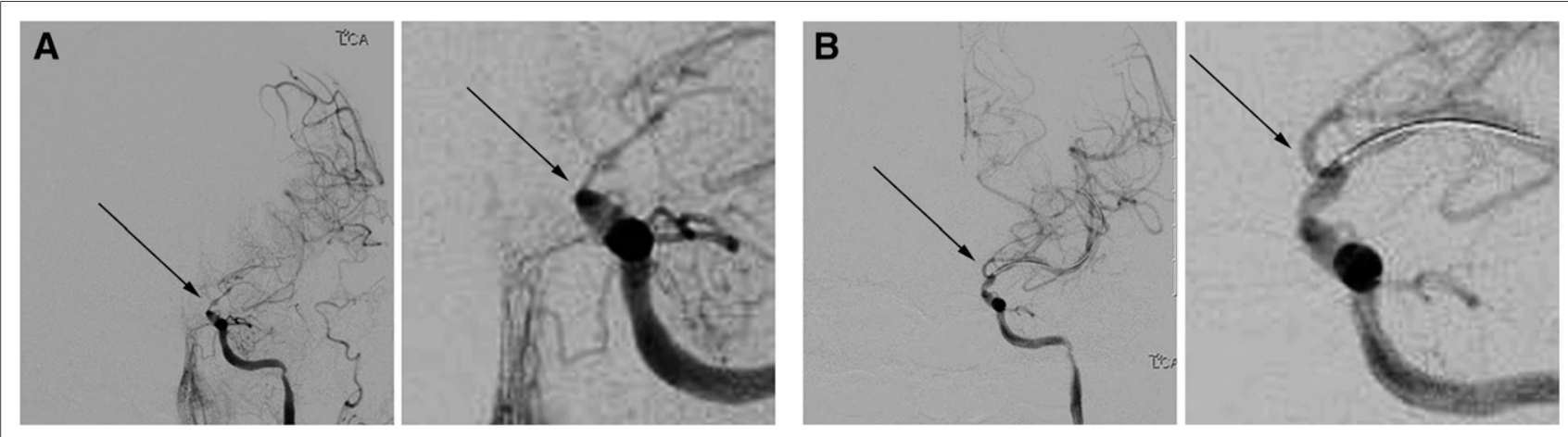

FIGURE 3. (A) LCA arteriogram images before LCA angioplasty (arrow shows flow limitation). (B) LCA arteriogram images after LCA angioplasty (arrow shows improved flow). LCA = left carotid artery.

\section{CASE DISCUSSION}

If the patient had not presented with an outside imaging diagnosis of intracerebral stenosis or with such a prodrome before acetazolamide SPECT imaging, other etiologies to include in a differential diagnosis might have been trauma, infection, and neoplasia. After imaging, other etiologies to consider were abnormal presentation of Kawasaki disease, hypertension, and migraine.

Acetazolamide SPECT imaging for neurologic vascular anomaly has been generally performed in many ways, ranging from a single-day split dose to a single-day dual-isotope $\left({ }^{99 \mathrm{~m}} \mathrm{Tc}\right)$ technique. Most studies use a 2-d challenge first-if the challenge is normal, the test is stopped. In this case, a $2-\mathrm{d}{ }^{99} \mathrm{~m} T \mathrm{Tc}-\mathrm{exametazine}$ baseline test was used first, followed by an 
acetazolamide technique. This procedure was not in accordance with current guidelines of the Society of Nuclear Medicine and Molecular Imaging, but it was supported by a unique clinical situation.

Acetazolamide SPECT imaging for a neurologic vascular anomaly is a valuable tool in a modern nuclear medicine department. Occlusion of the internal carotid artery carries a 30\% risk of ischemia of the ipsilateral cerebral hemisphere (4). Neither CT not MR imaging can fully define the physiologically significant flow reserve in a stenotic cerebral vessel. Regardless of the technique, the interpretation of SPECT and SPECT/CT neurologic imaging is user-dependent and can be fraught with pitfalls. Having a solid familiarity with normal regional and side-to-side variation is critical. ${ }^{99 \mathrm{~m}} \mathrm{Tc}-$ hexamethylpropyleneamine oxime imaging with carotid artery balloon occlusion has shown a discrepancy of at least $10 \%$ in side-to-side or regional radiotracer accumulation to be important (4).

\section{DISCLOSURE}

No potential conflict of interest relevant to this article was reported. The views expressed in this presentation are those of the author and do not necessarily reflect the official policy or position of the Department of the Navy, Department of Defense, or the U.S. government. This work was prepared as part of the author's official duties. Title 17, USC, §105 provides that "Copyright protection under this title is not available for any work of the U.S. Government." Title 17, USC, $\S 101$, defines a U.S. government work as a work prepared by a military service member or employee of the U.S. government as part of that person's official duties.

\section{REFERENCES}

1. Juni JE, Waxman AD, Devous MD, et al. Procedure guideline for brain death perfusion SPECT using 99mtTc radiopharmaceutical 3.0. 2009. Available at: http://www.snm. org/guidelines.

2. Acetazolamide. Available at: http://www.drugs.com/pro/acetazolamide.html.

3. Iida H, Nakagawara J, Hayashida K, et al. Multicenter evaluation of a standardized protocol for rest and acetazolamide cerebral blood flow assessment using a quantitative SPECT reconstruction program and split-dose 123-I-Iodoamphetamine. J Nucl Med. 2010;51:1624-1631.

4. Monsein LH, Jeffery PJ, van Heerden BB, et al. Assessing adequacy of collateral circulation during balloon test occlusion of the internal carotid artery with $99 \mathrm{~m}-\mathrm{Tc}-$ HMPAO SPECT. AJNR. 1991;12:1045-1051.

${ }^{*}$ For the answers, see page $10 \mathrm{~A}$. 